\title{
INTRODUCTION
}

Dear readers,

We are pleased to present Part 1 of 2 of Radiocarbon 2018, the Proceedings of the 23rd International Radiocarbon Conference, which was held June 17-22, 2018, in Trondheim, Norway and hosted by NTNU University Museum. Part 2 will be published in the December 2019 issue of Radiocarbon.

The study of radiocarbon is broad, so this meeting brought together researchers from many fields and interests. We are grateful to the more than 280 participants from around the world who met in Trondheim to share their diverse ideas and perhaps discuss new collaborations. We would like to thank the sponsors, invited speakers, and committee members for their invaluable contributions. We also thank all the participants for their engaging and fruitful discussions, both in session and out. In all, we hosted approximately 110 oral presentations and over 230 poster presentations.

When the sessions were done for the day, participants were free to explore the lovely town of Trondheim, which is within walking distance of the conference venue, the Scandic Lerkendal hotel. Excursions included pub nights as well as a beautiful organ concert in the historic Nidaros Cathedral, followed by a gala dinner held at the stately Frimurerlogen building, where guests were treated to live music, an excellent meal, wine, and (for some) dancing into the night.

We hope everyone enjoyed their experience in Trondheim and we hope to see you all again soon. The next Radiocarbon Conference will be held in 2021 in Israel, coordinated by Elisabetta Boaretto and hosted by the Weizmann Institute of Science.

On behalf of the Organizing Committee,

Marie-Josée Nadeau

Guest editor 


\section{ORGANIZING COMMITTEES}

\section{Local Organizing Committee}

Marie Josée Nadeau

Martin Seiler

Solveig Bakken

Pieter M. Grootes

John Øystein

Haarsaker

Sylvie Lélu

Sølvi Stene

Helene Løvstrand Svarva

Terje Thun

Einar Værnes

\section{Scientific Committee}

Philippa Ascough

Alex Bayliss

Elizabetta Boaretto

Mathieu Boudin

Lucio Calcagnile

Alexander E. Cherkinsky

Gordon Cook

Carley Crann

Michael Dee

Stewart Fallon

Pieter M. Grootes

Irka Hajdas

Christine Hatté

Alan Hogg

Quan Hua

Eiliv Larsen

Ann McNichol

John Meadows

Gesine Mollenhauer
Mihály Molnár

Marie-Josée Nadeau

Jesper Olsen

Gianluca Quarta

Andrzej Rakowski

Janet Rethemeyer

Guaciara dos Santos

Bettina Schulz-Paulsson

Linda Scott Cummings

Hongtao Shen

Andrew Smith

John Southon

Axel Steinhof

Kristina Stenström

Sönke Szidat

Susan Trumbore

Lukas Wacker

Eva-Maria Wild

Antoine Zazzo

\section{SPONSORS}

We want to thank our sponsors for their generous contributions:

The Research Council of Norway

The Norwegian University of Science and Technology

Det Kongelige Norske Videnskabers Selskab Ionplus

High Voltage Engineering

National Electrostatic Corporation

Thermo Fisher

Cambridge University Press 


\section{PHOTOS}

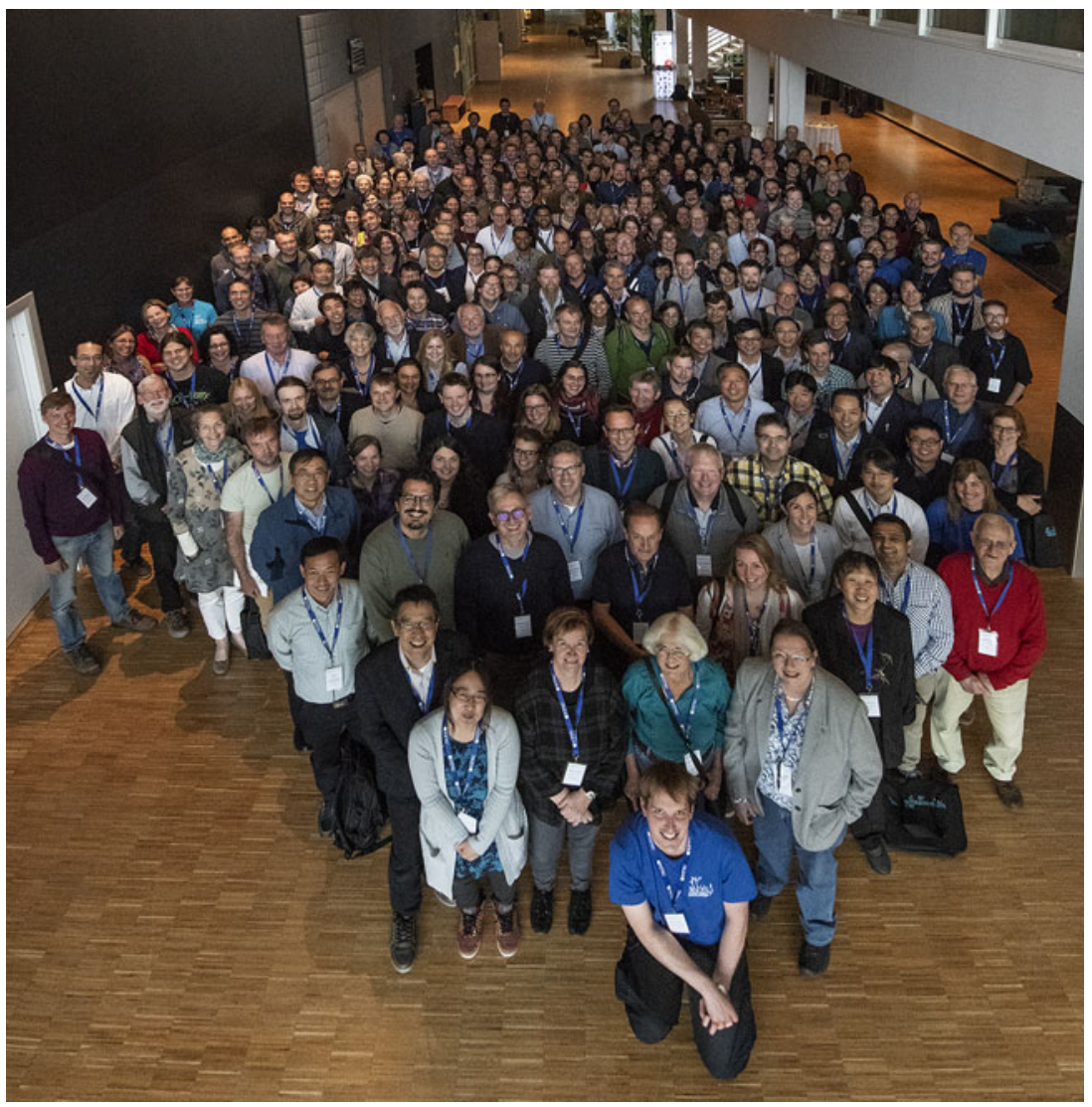

Participants of Radiocarbon 2018 at the Scandic Lerkendal conference center in Trondheim, Norway. (Photos courtesy of the organizers.)

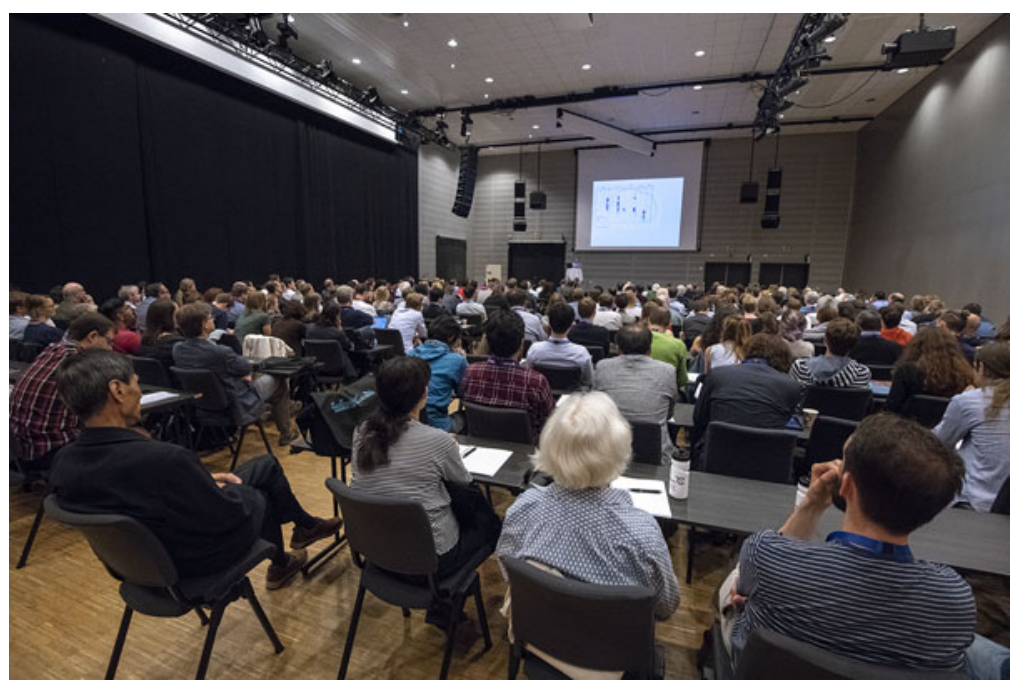

Conference participants listening to the opening plenary talk of Elisabetta Boaretto. 
viii

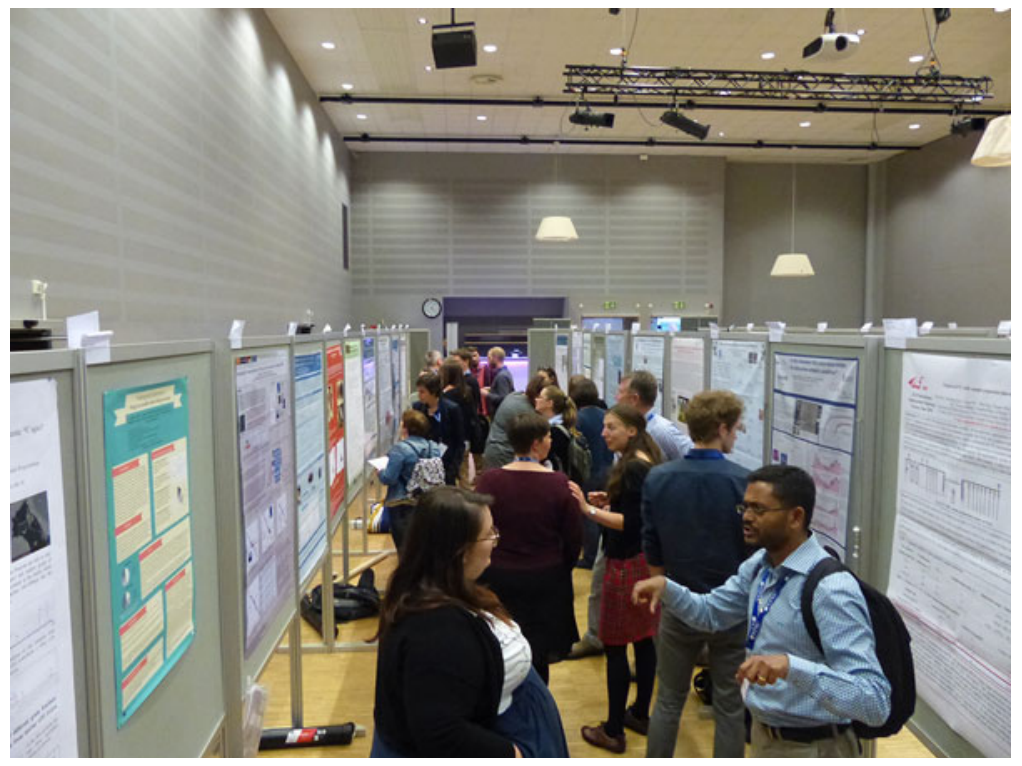

Discussions during a poster session.

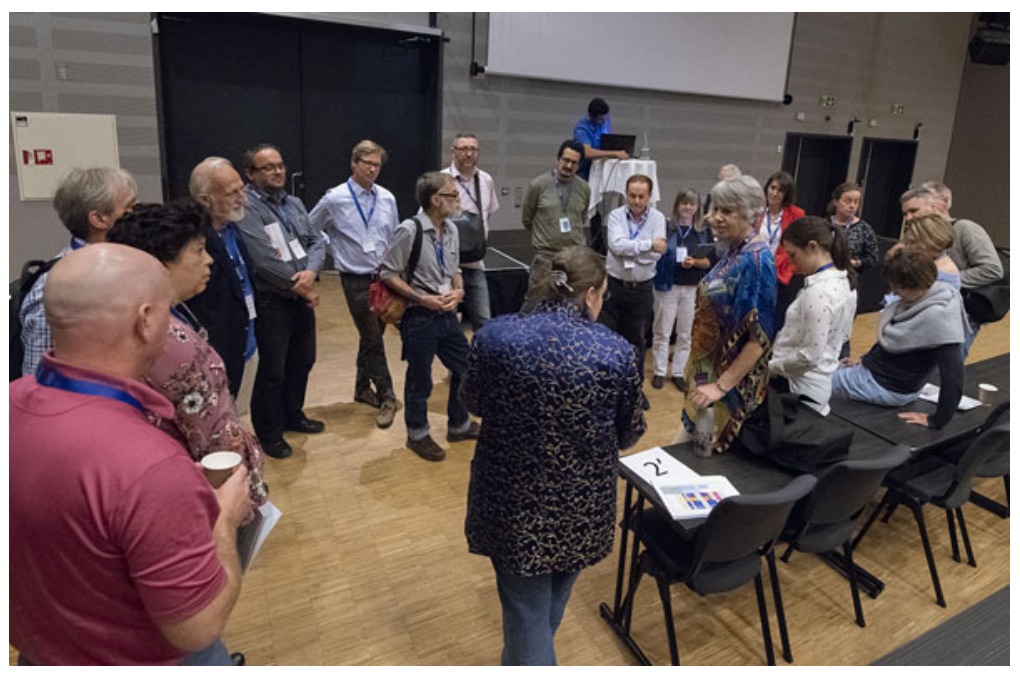

Gathering of the scientific committee on the first conference day. 


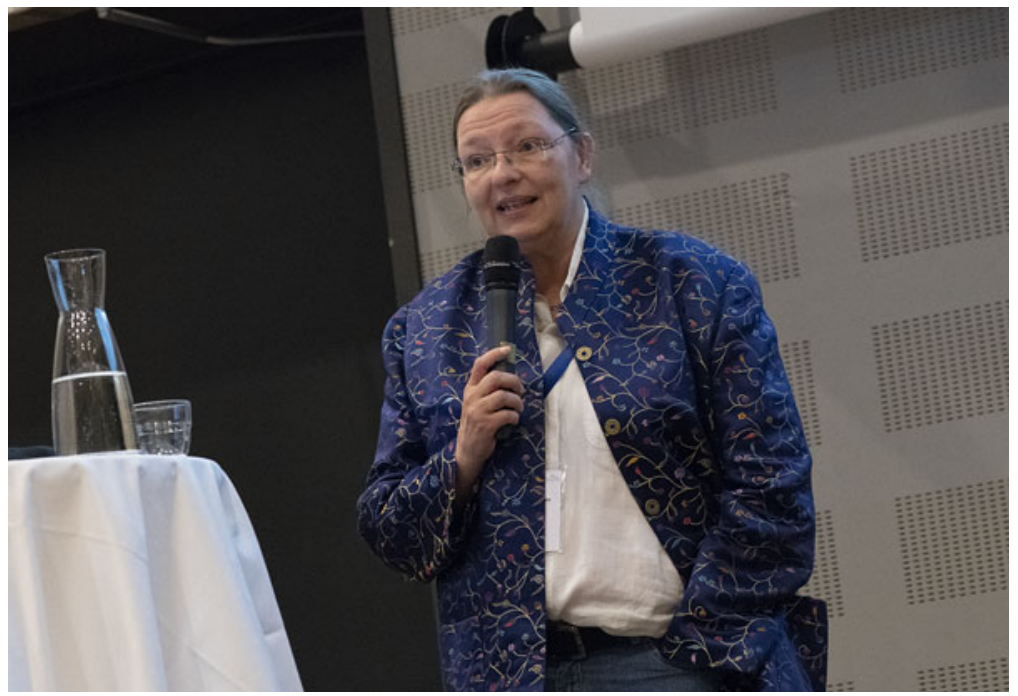

Conference host Marie-Josée Nadeau giving her opening speech for the Radiocarbon 2018 conference.

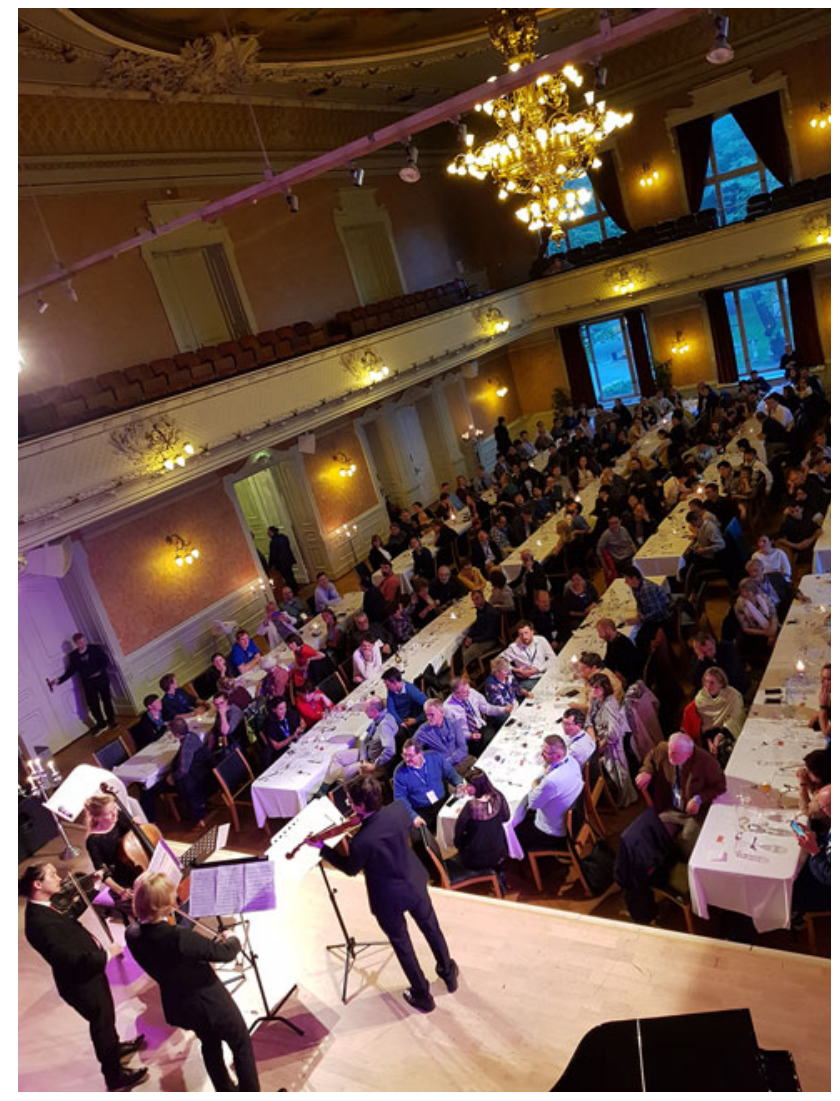

Bovin quartet performing at the gala dinner. 


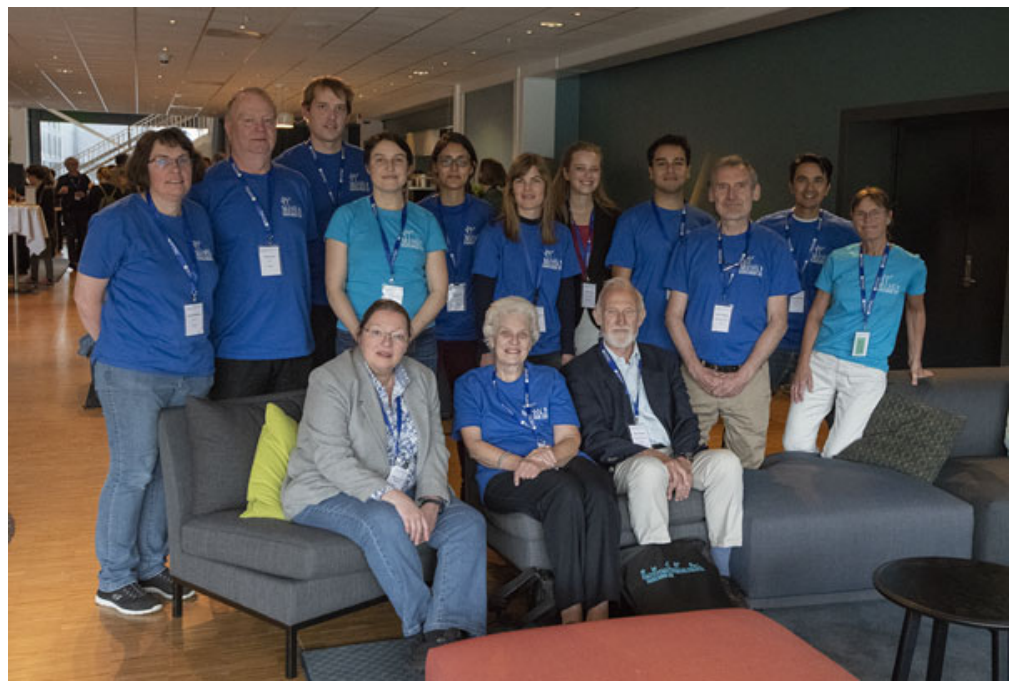

Local organizing committee from NTNU. 


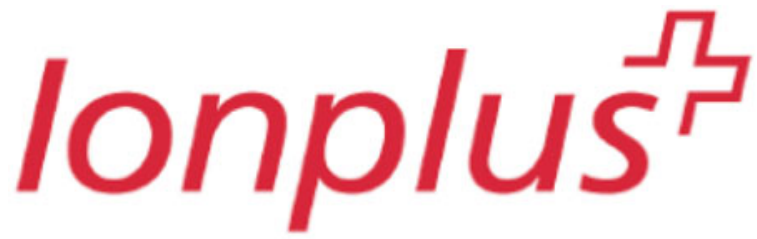
engineering scientific instruments

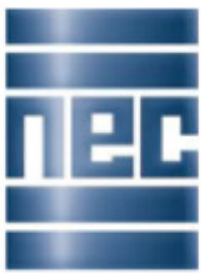

\section{National}

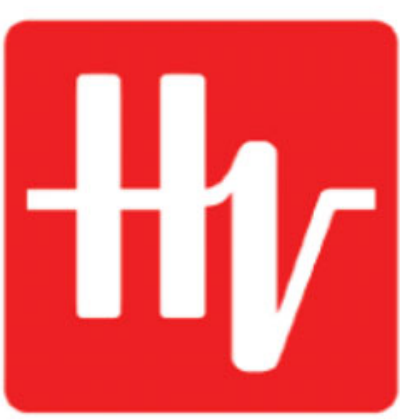

Electrostatics

Corp.

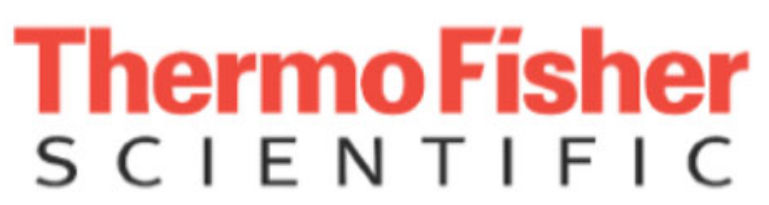

\section{圈 CAMBRIDGE}

UNIVERSITY PRESS

The Research Council of Norway
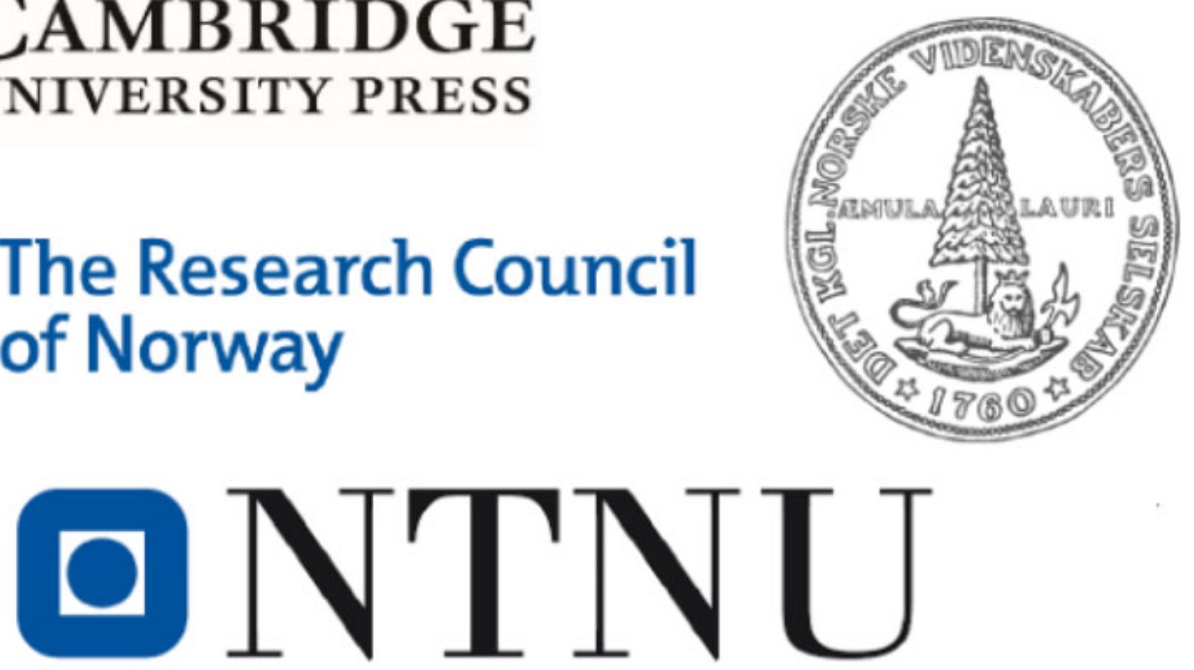
CONFERENCE PARTICIPANTS

\begin{tabular}{|c|c|c|c|}
\hline Anita & Aerts-Bijma & Kimberley & Elliott \\
\hline Kerry & Allen & Asya & Engovatova \\
\hline Eduardo & Alves & Christophe & Espic \\
\hline Takafumi & Aramaki & Žilvinas & Ežerinskis \\
\hline Philippa & Ascough & Simon & Fahrni \\
\hline Takahiro & Aze & Stewart & Fallon \\
\hline Solveig & Bakken & Ionut & Faurescu \\
\hline Edouard & Bard & Helen & Fewlass \\
\hline Serena & Barone & Keith & Fifield \\
\hline Gerard & Barrett & Kari & Finstad \\
\hline Peter & Barta & Alexandra & Fogtmann-Schulz \\
\hline Alex & Bayliss & Norbert & Frank \\
\hline Steven & Beaupre & Stewart & Freeman \\
\hline Lorena & Becerra Valdivia & Ronnie & Friedrich \\
\hline Lucille & Beck & Yunchong & $\mathrm{Fu}$ \\
\hline Lars & Beckel & Naoto & Fukuyo \\
\hline Jeffrey & Beem-Miller & Alan & Gagnon \\
\hline Elisabetta & Boaretto & Torben & Gentz \\
\hline Mathieu & Boudin & Andrea & Giannini \\
\hline Joël & Bourquin & Merle & Gierga \\
\hline Veronika & Brychova & Magnar Mojaren & Gran \\
\hline Botond & Buró & Grada & Grootes \\
\hline Martin & Butzin & Pieter & Grootes \\
\hline Manuela & Capano & Yongjing & Guan \\
\hline Israel & Carmi & John Øystein & Haarsaker \\
\hline Carla & Carvalho & Kirstine & Haase \\
\hline Emmanuelle & Casanova & Carla & Hadden \\
\hline Ingrid & Chanca & Negar & Haghipour \\
\hline Zhineng & Cheng & Irka & Hajdas \\
\hline Peng & Cheng & Karl & Håkansson \\
\hline Alexander E. & Cherkinsky & Christian & Hamann \\
\hline David & Chivall & Ronald & Hatfield \\
\hline Ian & Clark & Christine & Hatté \\
\hline Gordon & Cook & Ming & $\mathrm{He}$ \\
\hline Carley & Crann & Tim & Heaton \\
\hline Brendan & Culleton & Jonathan & Heile \\
\hline Linda & Cummings & Jan & Heinemeier \\
\hline Søren & Dalby & Laura & Hendriks \\
\hline Daniele & De Maria & Christopher & Hill \\
\hline Guy & De Mulder & Shoko & Hirabayashi \\
\hline Michael & Dee & Benjamin & Hmiel \\
\hline Emmanuelle & Delqué-Kolic & Gregory & Hodgins \\
\hline Thibaut & Deviese & Alan & Hogg \\
\hline Ping & Ding & Wan & Hong \\
\hline Fiona & Doessel & Rachel J.A. & Hopkins \\
\hline Kejun & Dong & Quan & Hua \\
\hline Guaciara & Dos Santos & Mathias & Huels \\
\hline Katerina & Douka & Narumi & Ishizawa \\
\hline Jean-Pascal & Dumoulin & Eileen & Jacob \\
\hline Elaine & Dunbar & Piotr & Jacobsson \\
\hline Timothy & Eglinton & Andrea & Jaeschke \\
\hline Maria & Eifrem & Christabel & Jean \\
\hline Kathryn & Elder & Hyeonyeol & Jeon \\
\hline
\end{tabular}




\begin{tabular}{|c|c|c|c|}
\hline A.J. Timothy & Jull & Mike & Mores \\
\hline Marie & Kanstrup & Toru & Moriya \\
\hline Kenny & Kearney & Anne & Mouchet \\
\hline Evelyn & Keaveney & Arnold & Müller \\
\hline Nicholas & Kessler & Sarah & Murseli \\
\hline Liam & Kieser & Raimund & Muscheler \\
\hline Matthias & Klein & Solene & Mussard \\
\hline Timothy & Knowles & Marie-Josée & Nadeau \\
\hline Peter & Köhler & Toshio & Nakamura \\
\hline Yoko & Kokubu & Toshimichi & Nakanishi \\
\hline Ivan & Kontul' & Philip & Naysmith \\
\hline Ines & Krajcar Bronić & Andreas & Neocleous \\
\hline Marek & Krapiec & Zhenchuan & Niu \\
\hline Bernd & Kromer & Jessica & Nordby \\
\hline Joel & Kronfeld & Hirotaka & Oda \\
\hline Kaoru & Kubota & Mitsuru & Okuno \\
\hline Sabrina G. K. & Kudsk & Jesper & Olsen \\
\hline Margot & Kuitems & Takayuki & Omori \\
\hline Pankaj & Kumar & Algirdas & Pabedinskas \\
\hline Dai & Kunikita & Sanne & Palstra \\
\hline Mark & Kurz & Junghun & Park \\
\hline Walter & Kutschera & Adrian & Patrut \\
\hline Guillaume & Labrecque & Roxanna & Patrut \\
\hline Marleen & Lausecker & Dipayan & Paul \\
\hline Jean-Claude & Lefevre & Charlotte & Pearson \\
\hline Sylvie & Lelu & Antto & Pesonen \\
\hline Vladimir & Levchenko & Rita & Peyroteo Stjerna \\
\hline Hong-Chun & $\mathrm{Li}$ & Anne & Philippe \\
\hline Lucia & Liccioli & Bente & Philippsen \\
\hline Susanne & Lindauer & Natalia & Piotrowska \\
\hline Qi & Liu & Pavel & Povinec \\
\hline Madison & Llewellin & Gurazada & Prasad \\
\hline Brett & Longworth & Katherine & Pugsley \\
\hline Kita & Macario & Gianluca & Quarta \\
\hline Istvan & Major & Anita & Quiles \\
\hline Sahib & Mammadov & Andrzej & Rakowski \\
\hline Rikke & Maring & Christopher & Ramsey \\
\hline Miguel Angel & Martinez Carrillo & Johanna & Regev \\
\hline Marc & Massault & Lior & Regev \\
\hline Tetsuya & Matsunaka & Paula J. & Reimer \\
\hline Hiroyuki & Matsuzaki & Pascale & Richardin \\
\hline Ann P & McNichol & Anke & Rieck \\
\hline Harro & Meijer & Mark & Roberts \\
\hline Jan Olaf & Melchert & Helene & Rose \\
\hline Cyrielle & Messager & Minoru & Sakamoto \\
\hline Andrew & Millard & Paula Utigard & Sandvik \\
\hline Masayo & Minami & Michael & Sarnthein \\
\hline Eugenia & Mintz & Kimikazu & Sasa \\
\hline Yosuke & Miyairi & Tiberiu & Sava \\
\hline Fusa & Miyake & Mikkel Fristrup & Schou \\
\hline Kenichiro & Mizohata & Bettina & Schulz Paulsson \\
\hline Thomas & Moffat & Andrea & Scifo \\
\hline Gesine & Mollenhauer & Marian & Scott \\
\hline Mihály & Molnár & Martin & Seiler \\
\hline Christophe & Moreau & Hongtao & Shen \\
\hline
\end{tabular}


xiv

$\begin{array}{llll}\text { Vasily } & \text { Shishkov } & \text { Irina } & \text { Vagner } \\ \text { Pavel } & \text { Simek } & \text { Tess } & \text { Van Den Brande } \\ \text { Margit } & \text { Simon } & \text { Johannes } & \text { Van Der Plicht } \\ \text { Andreja } & \text { Sironić } & \text { Henri } & \text { Van Oosterhout } \\ \text { Tina } & \text { Skjærvik Thomsen } & \text { Tamás } & \text { Varga } \\ \text { Andrew } & \text { Smith } & \text { R. A. } & \text { Varney } \\ \text { Nichla } & \text { Smith } & \text { Marie-Anne } & \text { Vibet } \\ \text { Corina } & \text { Solis } & \text { John } & \text { Vogel } \\ \text { Adam } & \text { Sookdeo } & \text { Lukas } & \text { Wacker } \\ \text { Guillaume } & \text { Soulet } & \text { Brett } & \text { Walker } \\ \text { John } & \text { Southon } & \text { Jennifer } & \text { Walker } \\ \text { Richard } & \text { Staff } & \text { Xuchen } & \text { Wang } \\ \text { Axel } & \text { Steinhof } & \text { Lyndelle } & \text { Webster } \\ \text { Sølvi } & \text { Stene } & \text { Caroline } & \text { Welte } \\ \text { Noah } & \text { Steuri } & \text { Eva Maria } & \text { Wild } \\ \text { Alexander } & \text { Stolz } & \text { Philipp } & \text { Wischhöfer } \\ \text { Mark } & \text { Sundquist } & \text { Rachel } & \text { Wood } \\ \text { Kilho } & \text { Sung } & \text { Feng } & \text { Xie } \\ \text { Helene Løvstrand } & \text { Svarva } & \text { Xiaomei } & \text { Xu } \\ \text { Árný Erla } & \text { Sveinbjörnsdóttir } & \text { Kazuhiro } & \text { Yagasaki } \\ \text { Ivo } & \text { Svetlik } & \text { Masako } & \text { Yamane } \\ \text { Soenke } & \text { Szidat } & \text { Yusuke } & \text { Yokoyama } \\ \text { Hiroshi } & \text { Takahashi } & \text { Qubo } & \text { You } \\ \text { Chiara } & \text { Telloli } & \text { Ingrid } & \text { Ystgaard } \\ \text { Filippo } & \text { Terrasi } & \text { Elya } & \text { Zazovskaya } \\ \text { Bruno } & \text { Thellier } & \text { Antoine } & \text { Zazzo } \\ \text { Steffen } & \text { Therre } & \text { Weijian } & \text { Zhou } \\ \text { Terje } & \text { Thun } & \text { Sanyuan } & \text { Zhu } \\ \text { Nadine } & \text { Tisnérat Laborde } & \text { Yizhi } & \text { Zhu } \\ \text { Masao } & \text { Uchida } & & \\ \text { Joonas } & \text { Uusitalo } & & \\ \text { Einar } & \text { Værnes } & & \\ & \text { Sal } & & \end{array}$




\section{ANNOUNCEMENT}
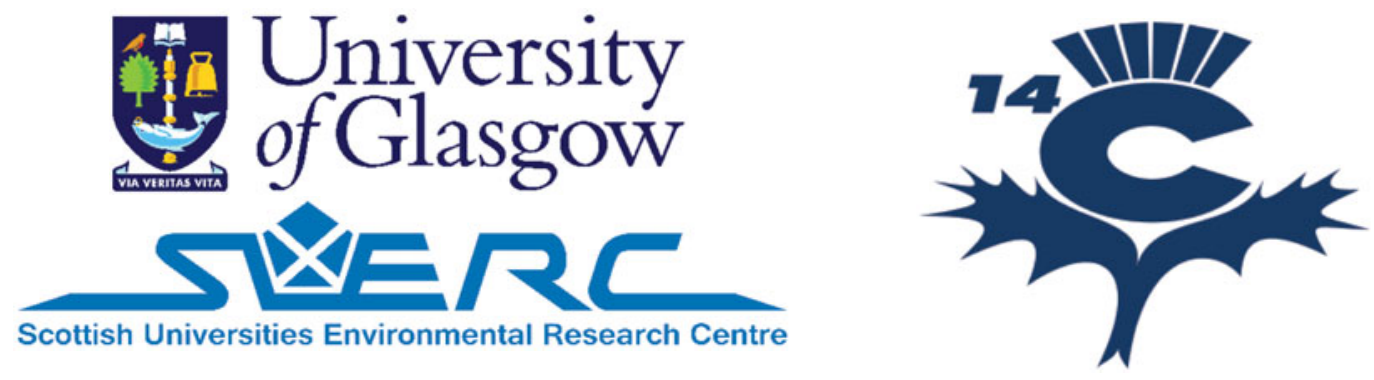

\section{Glasgow Radiocarbon Inter-Comparison 2019}

The next global radiocarbon inter-comparison is currently being planned, with samples being sourced. We will distribute the samples by the end of 2019, allowing 6 months for return of the results. This exercise is for AMS facilities, but we will endeavour, if possible to acquire radiometric samples.

\section{Samples:}

All of the samples are natural (wood, bone, peat and grain), some are known age, and overall their age spans approx. 40,000BP to modern. In the case of peat, the sample will have been pretreated to humic acid, but other samples will require pre-treatment.

We have designed a study with two groups of samples. The first group is typical of the samples provided in SIRI, where the volume of material being provided will be sufficient to make a few repeat measurements. In the second group, we will provide a quantity of material, sufficient to allow AMS labs to run (and report) multiple measurements from different wheels/batches over the space of six months experimental phase. It is intended that sufficient material will remain to allow labs to use these as internal quality assurance samples.

\section{Why this design?}

The purpose of including the first group of samples is to allow each laboratory to quality check (once consensus values and uncertainties have been defined), their laboratory operation at the time of analyses (so a classical round robin trial). The second group of samples provides laboratories with well characterised materials which can function as secondary standards, in sufficient quantity to be run routinely and thus allow assessment of both laboratory precision and accuracy.

\section{What should you do?}

If you are interested in taking part and receiving samples, then please email marian.scott@, glasgow.ac.uk or philip.naysmith@glasgow.ac.uk. 